\title{
The nature of the IR emission in LLAGN at parsec scales
}

\section{Does the jet dominate at low-luminosities?}

\author{
Juan Antonio Fernández-Ontiveros ${ }^{1, a}$, M. Almudena Prieto ${ }^{2,3}$, Jose Antonio Acosta-Pulido ${ }^{2,3}$, Sera Markoff ${ }^{4}$, and \\ Omaira González-Martín²,3 \\ ${ }^{1}$ Max-Planck-Institut für Radioastronomie (MPIfR), Auf dem Hügel 69, Bonn, D-53121, Germany \\ ${ }^{2}$ Instituto de Astrofísica de Canarias (IAC), Vía Láctea s/n, La Laguna, E-38200, Spain \\ ${ }^{3}$ Departamento de Astrofísica, Facultad de Física, Universidad de La Laguna, Astrofísico Fco. Sánchez s/n, La Laguna, E- \\ 38207, Spain \\ ${ }^{4}$ Astronomical Institute "Anton Pannekoek", 1098 XH Amsterdam, Netherlands
}

\begin{abstract}
The vast majority of AGN belong to the low-luminosity class (LLAGN): they exhibit a low radiation efficiency $\left(L \lesssim 10^{42} \mathrm{erg} \mathrm{s}^{-1} ; L / L_{\text {edd }} \lesssim 10^{-3}\right)$ and the absence of the big blue bump in their spectrum, a signature of the accretion disk. The study of LLAGN is a complex task due to the contribution of the host galaxy, whose light outshines these faint nuclei. As a consequence, numerical models are usually compared with relatively poorly defined spectral energy distributions (SEDs). For a sample of six prototype nearby LLAGN, a multiwavelength dataset including radio, IR, optical/UV and X-ray measurements with a few tenths of arcsec resolution has been collected. These high-spatial resolution SEDs reveal that: $i$ ) the mid-IR bump, indicative of thermal emission from the torus, is missing in LLAGN; ii) the continuum emission of these nuclei is largely described by a self-absorbed synchrotron spectrum, suggesting that jet emission dominates the overall energy output in these objects. The optically thin radiation in the IR-to-UV range is produced in the jet launching region, very close to the central black hole. The very steep slope found in this component -with a spectral index in the 1-3 range- suggests that a large number of LLAGN are powered by young and compact jets with very high radiative losses.
\end{abstract}

\section{Introduction}

The majority of AGN exhibit a low level of nuclear activity, characterised by a low accretion rate and a modest luminosity. These are known as low-luminosity AGN (LLAGN), the faintest and most numerous members of this family, which includes $\sim 1 / 3$ of all galaxies in the Local Universe [1]. However, LLAGN are not just scaledown versions of their bright counterparts, i.e. Seyfert galaxies and quasars. Their main characteristics are: $i$ ) absence of the big blue bump [2], signature of the accretion disk in the Spectral Energy Distribution (SED); ii) most are "radio-loud", showing compact cores and parsecscale radio jets [3]; and iii) they are frequently associated with low-ionisation nuclear emission-line regions (LINERs, [4]).

The differences between bright AGN and LLAGN are likely associated with the structural changes predicted at low luminosities: both the broad-line region and the "torus", keystones of the Unified Model [5], are expected to vanish at $L_{b o l} \lesssim 10^{42} \mathrm{erg} / \mathrm{s}[6,7]$. The paradigm is that, despite their low luminosity, LLAGN are in many aspects alike to Quasars: they are radio loud, often reside in massive ellipticals and usually display extraordinary jets. However, their spectral energy distributions

\footnotetext{
ae-mail: jafo@mpifr.de
}

(SEDs) are markedly different. The major known problem, the absence of the blue bump emission, led to the widely use of the advection dominated accretion solution ADAF models- successfully applied to X-ray binaries and in $\mathrm{Sgr} \mathrm{A}^{*}$ [8], to also explain LLAGN spectra and in turn their low luminosity [9-12]. Yet, the application of these models are based on relatively poorly defined SEDs due to insufficient spatial resolution and/or wavelength coverage. In particular, low resolution data in the IR -and thus, upper limits in this range- are common reference in the SEDs, since the host galaxy usually outshines these faint nuclei. Spatial resolution in these cases really matters to avoid being dominated by the stellar light.

\section{High-spatial resolution data}

The basis of this work is a multiwavelength, high-spatial resolution dataset that covers multiple spectral windows -radio, IR, optical/UV and X-rays- for the inner few kiloparsecs of a sample of six LLAGN. In particular, the use of Adaptive Optics (AO) in the near-IR (NACO) and diffraction limited imaging in the mid-IR (VISIR) at the Very Large Telescope (VLT) allowed us to correctly fill the gap in the $1-20 \mu \mathrm{m}$ region with truly nuclear measurements for the brightest and nearest LLAGN. The dataset was completed with the highest-spatial resolution data available in 
Table 1. Sample of galaxies sorted by increasing distance. The spatial resolution is estimated as the FwHM of the most compact object found in the FOV. $L_{\mathrm{bol}}$ is estimated from the high-spatial resolution SEDs.

\begin{tabular}{lccccll}
\hline Name & $\begin{array}{c}\text { D } \\
{[\mathrm{Mpc}]}\end{array}$ & Ref. & $\begin{array}{c}\text { FWHM } \\
{[\mathrm{pc}]}\end{array}$ & $\begin{array}{c}\mathbf{1}^{\prime \prime} \\
{[\mathrm{pc}]}\end{array}$ & Type & Class \\
\hline NGC 4594 & 9.08 & {$[13]$} & 3.1 & 44.0 & SA(s)a & LINER 2 \\
NGC 1097 & 14.2 & {$[14]$} & 8.9 & 68.8 & SB(r'1)b & LINER 1 \\
NGC 1386 & 15.3 & {$[13]$} & 6.7 & 74.2 & SB(s)a & Sy 2 \\
M87 & 16.7 & {$[15]$} & 9.2 & 81.0 & cD & LINER 1 \\
NGC 1052 & 18.0 & {$[13]$} & 10.5 & 87.3 & E4 & LINER 1.9 \\
NGC 3169 & 24.7 & {$[16]$} & 10.9 & 119.7 & SA(s)a & LINER 2
\end{tabular}

the optical/UV range from the Hubble Space Telescope (HST) scientific archive. Nuclear fluxes were measured using aperture photometry of the unresolved component in the centre $\left(\lesssim 0^{\prime} 1\right)$, subtracting the local background around $\left(0,2-0{ }^{\prime} 3\right)$ in order to remove the contribution of the host galaxy. In the mid-IR, PSF photometry was performed (see [17]). Measurements in the radio and X-ray ranges have been collected after an extensive and careful search in the literature. These correspond mainly to Very Large Array (VLA) and Very Long Baseline Interferometry (VLBI) in radio and Chandra, XMM-Newton and Integral at X-rays. Despite of their lower spatial resolution, the flux at high energies is dominated by the AGN and not by the host galaxy, and thus can be consistently compared with subarcsec measurements at other wavelength ranges. The characteristics of this dataset allow us to build, for each LLAGN in the sample, a consistent SED of the same physical region, very well sampled over a wide range in wavelength. Additionally, a low-spatial resolution SED based on apertures larger than few arcsec was also built using the NED database ${ }^{1}$ and measurements published in the literature. The low-spatial resolution data is complementary to the subarcsec SED, as they permit to identify those spectral ranges in which the host galaxy shows a strong contribution, that might contaminate the nuclear spectrum.

This project is a follow-up of The central parsecs of the nearest galaxies ${ }^{2}[17,18]$, a high-spatial resolution study of the brightest and nearest Seyfert galaxies carried out at subarcsec scales with the VLT. The objects included in the present work (Table 1) correspond to AGN one to two order of magnitude fainter selected among the brightest and nearest LLAGN accessible from the Southern Hemisphere. Their nuclei are not covered by dust lanes (except NGC 3169), thus the absence of the blue bump is genuine. Three of the objects are the canonical reference for definition of the LLAGN class (NGC 1052 in [4]; NGC 1097 in [19]; M87 in [9]). The sample also represents the lowluminosity class in terms of host galaxy (S0, E and Sa: e.g. M87, NGC 1052 and NGC 3169) and radio loudness/quietness (e.g. M87 vs. NGC 1097).

\subsection{The nature of the IR emission}

The multiwavelength high-spatial resolution SED allow us to: $i$ ) probe the genuine SED of the LLAGN; and

\footnotetext{
${ }^{1}$ http://ned.ipac.caltech.edu/

${ }^{2}$ http://www.iac.es/project/parsec/
}

ii) identify, for each object, the physical mechanisms that dominate the energy output in different wavelength ranges. Fig. 1 shows the subarcsec SEDs for the sample of LLAGN (black dots), sorted by increasing distance. Low-spatial resolution data (grey spikes) trace the contribution of the host galaxy, which dominates the overall emission in most of the cases. For comparison, the radioloud quasar template from [20] is also shown (orange-solid line). A self-absorbed synchrotron model (see below, [21]) has been fitted to the radio-to-UV range for each subarcsec SED (dashed line).

\subsection{LLAGN at parsec scales}

Three main characteristics are common for all the LLAGN in the sample (see Fig. 1): i) a flat radio continuum, more similar to radio-loud quasars rather than Seyfert nuclei; ii) mid-IR and X-ray ranges drive the bolometric luminosity of these nuclei; and iii) subarcsec SEDs present a non-thermal behaviour, showing a flat/inverted radio/IRto-optical spectrum characteristic of jet emission. We also find that the inverted IR-to-optical continuum does not follow the classical synchrotron cooling slope of about $\alpha \approx 0.7\left(S_{v} \propto v^{-\alpha}\right)$ but has a much steeper spectrum, in the $1<\alpha<3$ range. The Sombrero galaxy (NGC 4594) shows a soft thermal bump at $\sim 1 \mu \mathrm{m}$ that might be caused by contamination from the host galaxy light.

\subsection{An accretion disk and/or a torus?}

LLAGN do not seem to fit in the standard accretion scenario, showing significantly different SEDs when compared with quasars and Seyfert galaxies. This has been probed also for high-spatial resolution data $[18,22]$. The torus, if present, is not evidenced by the continuum emission which seems to follow a non-thermal distribution (Fig. 1). Radio-loud quasars are more similar to LLAGN in the radio and X-ray ranges, but the absence of a big blue bump in the latter suggest important structural differences between both classes. In contrast, recent studies based on the spectral slopes measured between optical/Xrays and mid-IR-X-rays reveal that LLAGN present values in agreement their bright counterparts [23,24]. These pinpoint to the presence of a classical accretion disk and a torus, respectively. In Fig. 2 we probe this behaviour for the best sampled objects, i.e. NGC 4594, NGC 1097, M87 and NGC 1052. In blue, we show the optical/X-ray 

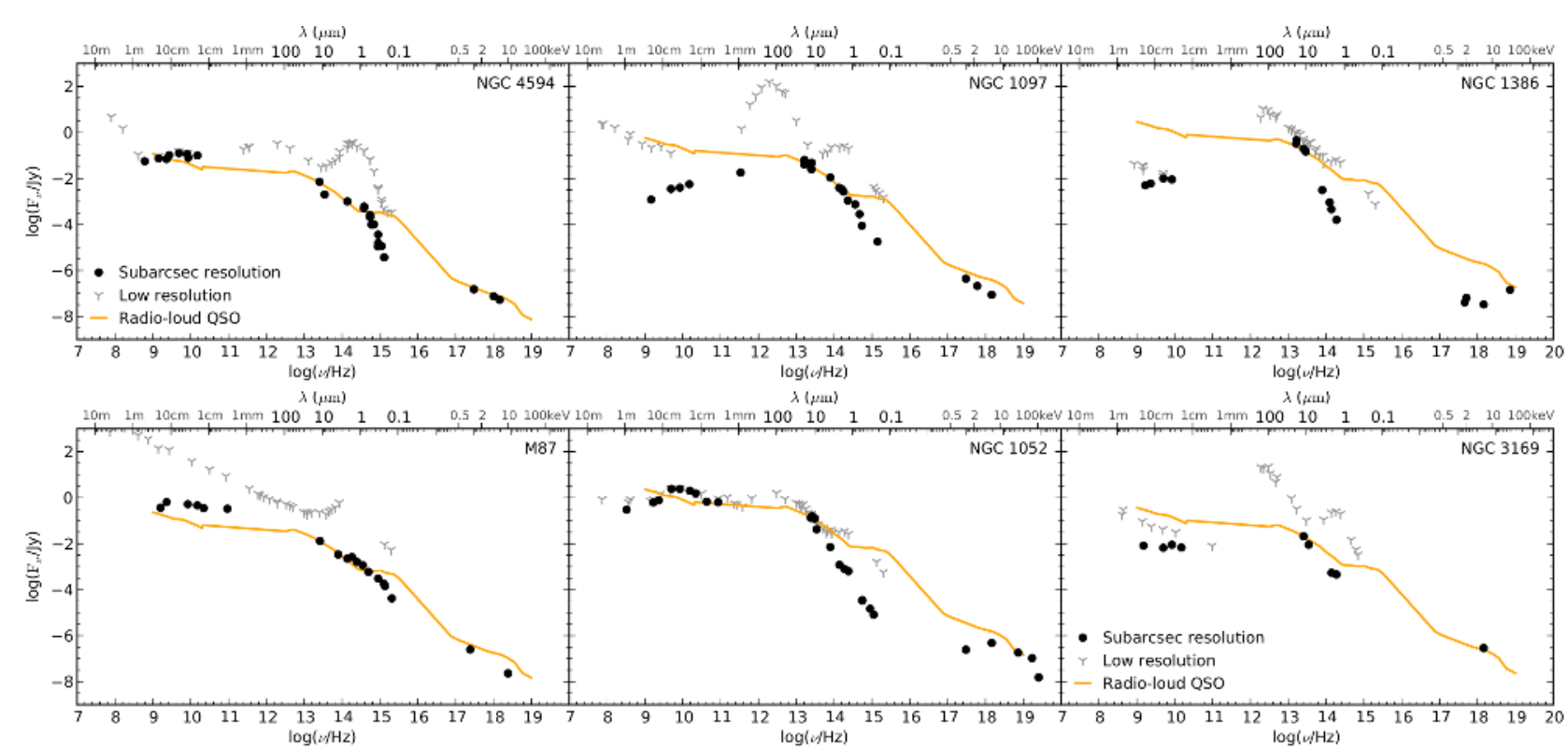

Figure 1. Subarcsec SEDs (black dots) and low-spatial resolution data (grey spikes) for the sample of LLAGN. The orange line corresponds to the radio-loud quasar template from [20]. A self-absorbed synchrotron model, i.e. a jet, has been fitted to the radio, IR and optical/UV ranges (dashed-line).

ratio found by [23] for a sample of LINERs, which have a spectral index $\alpha_{O X}$ between $2500 \AA$ and $2 \mathrm{keV}$ in the $0.8<\alpha_{O X}<1.4$ range $\left(S_{v} \propto v^{-\alpha_{O X}}\right)$. In each case, the most favourable case within this range is plotted. In red, we show the mid-IR to X-ray correlation found by [24] for a sample of nearby AGN, established between the flux at $12 \mu \mathrm{m}$ and the $2-10 \mathrm{keV} \mathrm{X}$-ray flux.

As shown by Fig. 2, the LLAGN in our sample are in agreement with the results from [23, 24]. However, this behaviour is not produced by thermal features in the SED. In contrast, both spectral slopes are produced by a steep power-law continuum from the mid-IR to optical/UV wavelengths. The non-thermal behaviour can be well described, at first order, by a self-absorbed synchrotron model [21] (dashed line in Fig. 2), suggesting a jet as the component that drives the overall continuum emission in these objects. In Section 3 we further explore this possibility using a more detailed model of a jet. Nonetheless, one should keep in mind that these spectral ratios are not able to discriminate bright AGN with strong thermal contributions in the mid-IR (torus) and the optical/UV (accretion disk) from LLAGN dominated by a steep non-thermal continuum as those included in our work.

\section{Inflow and Outflow}

ADAF and jet models in the literature are usually based on relatively poorly defined SEDs with insufficient spatial resolution and/or wavelength coverage. In particular, low-resolution data in the IR and, thus, upper limits in this range, are common reference. Our subarcsec SEDs allow us to probe the models in this range. Left panel in Fig. 3 shows an ADAF fit for the case of NGC 1052 (in black, [25]). The spectral features predicted by ADAF models, i.e. bumps in the millimetric to X-ray range, are not detected in any of the LLAGN of the sample. The mid-IR to optical/UV continuum in NGC 1052 largely departs from the ADAF prediction, following a very steep power law distribution $(\alpha=2.7)$. Such a high slope suggests that leptons in the base of the jet are cooling very fast due to high radiative losses. Alternatively, a very steep spectral index can be explained by a thermal lepton distribution ([26]; see also the talk by D. M. Russell). The right panel in Fig. 3 shows a fit of the jet+disk dominated model developed by S. Markoff [27, 28] to the SED of M87. Data for M87 are not simultaneous but are carefully selected from quiescent periods in which the core was in a low state of activity. The jet emission can explain the overall shape of the continuum emission for this LLAGN. Still, there are gaps in the SED that should be sampled (e.g. the submillimetric range with ALMA) in order to test the validity of these models and constrain the large number of parameters involved in the fit.

\section{Summary}

The importance of LLAGN lies in the fact that they permit to explore the limits of the classical picture for active nuclei. At very low luminosities the torus is expected to vanish and the classical accretion scenario changes, giving way to a radiatively inefficient structure. As a consequence of the changes in the inner structure, the SEDs of LLAGN present important differences with regard to the bright class. Subarcsec SEDs collected for a sample of six nearby LLAGN permit us to avoid the contamination from the host galaxy light and directly test the processes that dominate the emission for these objects. Despite of their host galaxies, the LLAGN in our sample show remarkably homogeneous high-spatial resolution SEDs, in 

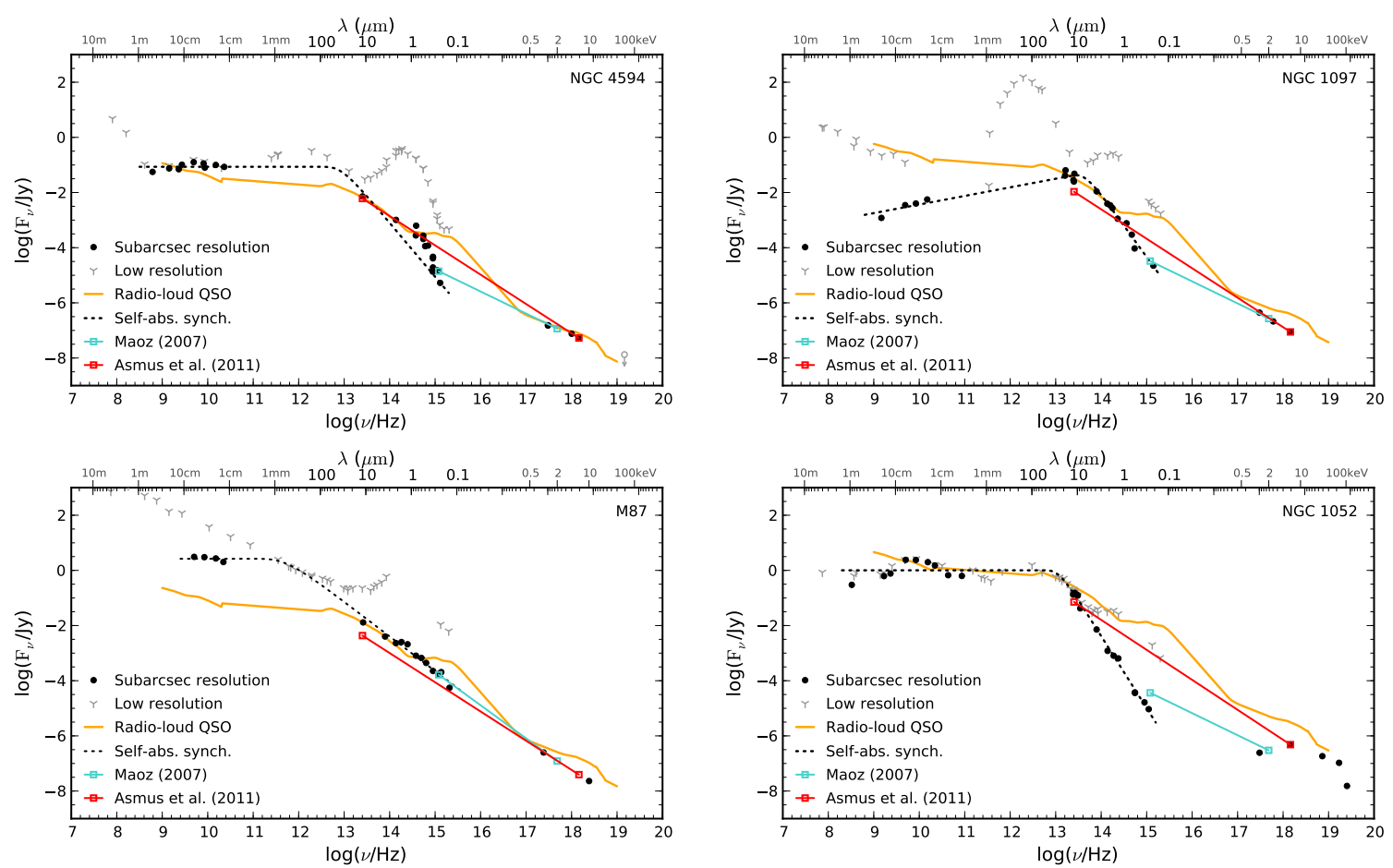

Figure 2. SEDs for the best sampled LLAGN: NGC 4594, NGC 1097, M87 and NGC 1052 from Fig. 1. In blue, the $2500 \AA 2 / 2 \mathrm{keV}$ ratio from [23]. In red, the $12 \mu \mathrm{m}$ to $2-10 \mathrm{keV}$ flux correlation found by [24].
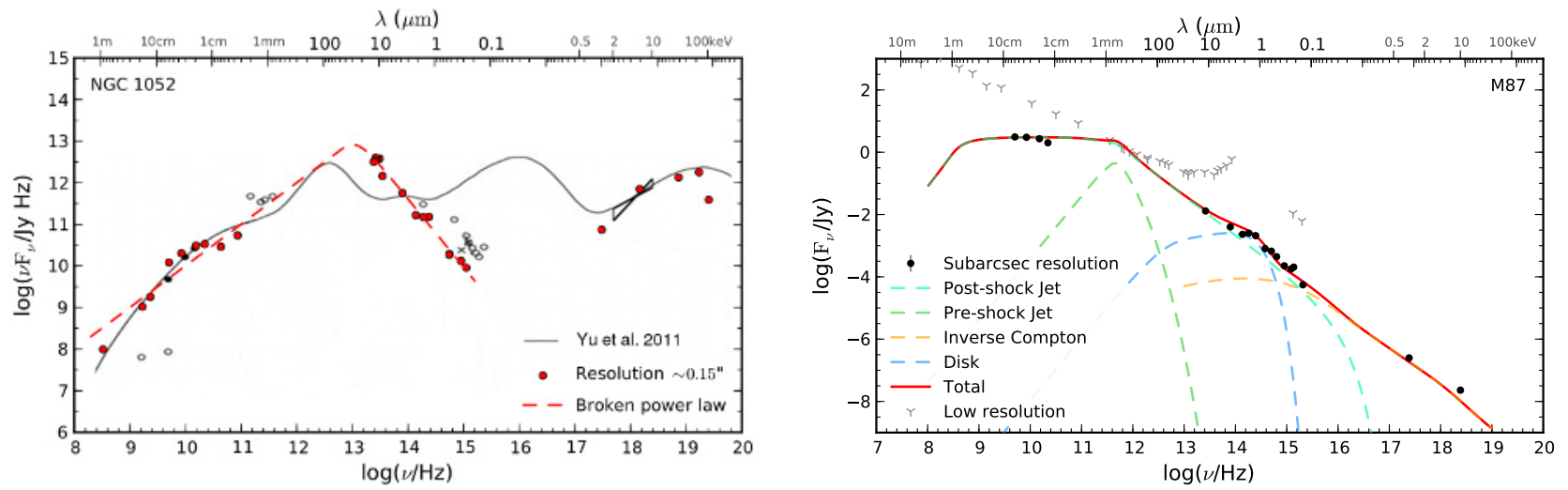

Figure 3. Left: ADAF model applied to NGC 1052 (in black, [25]), subarcsec SED collected in this work (red dots), and the selfabsorbed synchrotron model (red-dashed line). Right: jet+disk dominated model from [27, 28], applied to the subarcsec SED of M87. Individual components of the model are also plotted.

terms of their featureless shape. The torus and the accretion disk, if present, are not detected in the subarcsec SEDs. Most of the nuclei in the sample are well described by a flat/inverted radio/IR-to-optical continuum favouring the outflow scenario, i.e. a compact jet is driving the overall energy output. However, the inverted IR-to-optical continuum does not follow the classical synchrotron cooling slope and show a much steeper spectrum $(1<\alpha<3)$. Very high radiative losses or a thermal lepton distribution at the base of the jet can explain this behaviour.

\section{References}

[1] L.C. Ho, ARA\&A46, 475 (2008), 0803.2268
[2] L.C. Ho, PASP108, 637 (1996)

[3] N.M. Nagar, H. Falcke, A.S. Wilson, A\&A435, 521 (2005), arXiv: astro-ph/0502551

[4] T.M. Heckman, A\&A87, 152 (1980)

[5] R. Antonucci, ARA\&A31, 473 (1993)

[6] A. Laor, ApJ590, 86 arXiv: astro-ph/0302541

[7] S.F. Hönig, T. Beckert, MNRAS380, 1172 (2007)

[8] R. Narayan, I. Yi, R. Mahadevan, Nature374, 623 (1995)

[9] A.C. Fabian, M.J. Rees, MNRAS277, L55 (1995), arXiv: astro-ph/9509096 
[10] T. Di Matteo, S.W. Allen, A.C. Fabian, A.S. Wilson, A.J. Young, ApJ582, 133 (2003), arXiv: astro-ph/0202238

[11] F. Yuan, Z. Yu, L.C. Ho, ApJ703, 1034 (2009), 0902.3704

[12] M. Eracleous, J.A. Hwang, H.M.L.G. Flohic, ApJS187, 135 (2010), 1001. 2924

[13] J.B. Jensen, J.L. Tonry, B.J. Barris, R.I. Thompson, M.C. Liu, M.J. Rieke, E.A. Ajhar, J.P. Blakeslee, ApJ583, 712 (2003), arXiv: astro-ph/0210129

[14] R.B. Tully, L. Rizzi, E.J. Shaya, H.M. Courtois, D.I. Makarov, B.A. Jacobs, AJ138, 323 (2009)

[15] J.P. Blakeslee, A. Jordán, S. Mei, P. Côté, L. Ferrarese, L. Infante, E.W. Peng, J.L. Tonry, M.J. West, ApJ694, 556 (2009), 0901. 1138

[16] K.S. Mandel, W.M. Wood-Vasey, A.S. Friedman, R.P. Kirshner, ApJ704, 629 (2009), 0908.0536

[17] J. Reunanen, M.A. Prieto, R. Siebenmorgen, MNRAS402, 879 (2010), 0911. 2112

[18] M.A. Prieto, J. Reunanen, K.R.W. Tristram, N. Neumayer, J.A. Fernandez-Ontiveros, M. Orienti, K. Meisenheimer, MNRAS402, 724 (2010), Q910. 3771

[19] W.C. Keel, ApJ269, 466 (1983)
[20] M. Elvis, B.J. Wilkes, J.C. McDowell, R.F. Green, J. Bechtold, S.P. Willner, M.S. Oey, E. Polomski, R. Cutri, ApJS95, 1 (1994)

[21] A.P. Marscher, W.K. Gear, ApJ298, 114 (1985)

[22] R.E. Mason, E. Lopez-Rodriguez, C. Packham, A. Alonso-Herrero, N.A. Levenson, J. Radomski, C. Ramos Almeida, L. Colina, M. Elitzur, I. Aretxaga et al., AJ144, 11 (2012), 1205.0029

[23] D. Maoz, MNRAS377, 1696 (2007), arXiv: astro-ph/0702292

[24] D. Asmus, P. Gandhi, A. Smette, S.F. Hönig, W.J. Duschl, A\&A536, A36 (2011), 1109. 4873

[25] Z. Yu, F. Yuan, L.C. Ho, ApJ726, 87 (2011), 1011.1962

[26] T. Shahbaz, D.M. Russell, C. Zurita, J. Casares, J.M. Corral-Santana, V.S. Dhillon, T.R. Marsh, MNRAS434, 2696 (2013), 1307.0659

[27] S. Markoff, M.A. Nowak, J. Wilms, ApJ635, 1203 (2005), arXiv: astro-ph/0509028

[28] S. Markoff, M. Nowak, A. Young, H.L. Marshall, C.R. Canizares, A. Peck, M. Krips, G. Petitpas, R. Schödel, G.C. Bower et al., ApJ681, 905 (2008), 0804.0344 